\title{
The assessment of the integrated antioxidant system of the body and the phenomenon of spa reaction in the course of radon therapy: A pilot study
}

\author{
Jadwiga M. Kuciel-Lewandowska ${ }^{1, A, C, D}$, Lilla Pawlik-Sobecka ${ }^{2, B}$, Sylwia Płaczkowska ${ }^{2, B}$, \\ Izabela Kokot ${ }^{2, B, C}$, Małgorzata Paprocka-Borowicz ${ }^{1, A, E, F}$ \\ ${ }^{1}$ Department of Physiotherapy, Wroclaw Medical University, Poland \\ ${ }^{2}$ Department of Laboratory Diagnostics, Wroclaw Medical University, Poland \\ A - research concept and design; B - collection and/or assembly of data; C - data analysis and interpretation; \\ $D$ - writing the article; $E$ - critical revision of the article; $F$ - final approval of the article
}

Address for correspondence

Małgorzata Paprocka-Borowicz

E-mail:malgorzata.paprocka-borowicz@umed.wroc.pl

\section{Funding sources}

The study was financed within the framework

of the Polish Radon Cluster.

Conflict of interest

None declared

Received on November 12, 2016

Reviewed on December 6, 2016

Accepted on March 13, 2017

\begin{abstract}
Background. Spa reaction is an adaptive response of an organism, emerging as a result of external factors. It is a positive element of spa therapy leading to metabolic changes, which are important for the body. The effect of these changes is an increase in immune activity and regenerative reactions of the body. The mechanism of the response is not fully known.
\end{abstract}

Objectives. The aim of the study was to evaluate the changes observed in the field of the integrated antioxidant system of the body in the course of radon therapy, especially in reference to spa reaction.

Material and methods. The study was conducted in the health resort in Świeradów-Zdrój. The observation regarded patients undergoing treatment with radon water. Before the treatment, after 5 and 18 days of treatment, the total antioxidant status (TAS) was evaluated with the use of a standard colorimetric assay. The study group consisted of 35 patients with degenerative joints and disc disease. The control group consisted of 15 people selected from the employees of the spa, also suffering from osteoarthritis, who did not undergo radon therapy (without contact with radon).

Results. On the $5^{\text {th }}$ day of the treatment, in both groups, the TAS increase was observed with significantly worse results in the control group. After the treatment, in the study group, an increase in TAS was observed, whereas in the control group, a significant decrease in the TAS concentration was noted.

Conclusions. A beneficial effect of radon treatments on the growth of TAS in the body of the patients treated in the spa was demonstrated. The increase in the TAS concentration on the $5^{\text {th }}$ day of treatment may indicate the relationship between these changes and the phenomenon of spa response. The changes are a result of low doses of ionizing radiation originating from radon dissolved in medicinal water, used in the course of the therapy.

Key words: balneotherapy, radiation hormesis, total antioxidant status, radon water, spa reaction

DOI

10.17219/acem/69450

\section{Copyright}

Copyright by Author(s)

This is an article distributed under the terms of the

Creative Commons Attribution Non-Commercial License

(http://creativecommons.org/licenses/by-nc-nd/4.0/) 


\section{Introduction}

In living organisms, oxygen reacts with organic compounds oxidizing them without undergoing total reduction due to the action of various, both external and internal, factors. The consequence of these shortcomings is the formation of reactive oxygen species (ROS). Among ROS, which are also called free radicals, there can be: singlet oxygen ${ }^{1} \mathrm{O}_{2}$, superoxide anion, hydrogen peroxide $\mathrm{H}_{2} \mathrm{O}_{2}$, and a hydroxide radical $\mathrm{OH}$ responsible for intracellular damage and, in consequence, apoptosis. In the state of health, the level of free radicals is strictly controlled in the process of maintaining a balance between their generation and removal. ${ }^{1-3}$ The generation of ROS or decreased possibility to remove free radicals is defined as oxidative stress. ${ }^{4,5}$ The consequence of these effects is cell damage and the development of many diseases, such as atherosclerosis, Alzheimer's disease, Parkinson's disease, inflammation, degenerative diseases of the skeletal system, allergies, cancer, diabetes, macular degeneration. ${ }^{6-8}$ The human body can be protected from the harmful effects of ROS by producing various metabolic reactions, both regulating and blocking the production of free radicals. The antioxidant defense system includes:

- endogenous antioxidants - produced by the body:

- enzymatic - antioxidant enzymes: superoxide dismutase, glutathione peroxidase, catalase;

- non-enzymatic: linolenic acid, polyamides, albumin, bilirubin, glutathione, uric acid, ceruloplasmin, transferrin, coenzyme Q10 (these compounds have different points of binding) $;{ }^{9}$

- exogenous antioxidants - delivered from the outside: vitamins C, A, E, carotenoids, xanthophylls, polyphenols.

These compounds are directly involved in free radical reactions; they affect both the transmission of cellular signals and the activity of enzymes or genes involved in the processes of cell death and DNA repair. ${ }^{10-12}$

The evaluation of the antioxidant system can be performed with the use of a variety of methods, by assessing the total antioxidant status (TAS) or its individual components, especially in the first studied group. The present study concerned the total antioxidant capacity of plasma, TAS. The integrated antioxidant system embracing all biological components active in preventing oxidation was assessed.

Radon therapy in Europe has been used for over 100 years. Obviously, therapeutic programs were modified throughout the last century due to actively developing hydrotherapy and spa therapy. In Polish conditions, radon therapy can be ordained medically only by a doctor after considering the indications and contraindications, and then the standard number of treatments is prescribed. The number of treatments, their duration and type, such as inhalations, baths or mouthwashes (especially periodontal rinses) are defined.
Radon health resorts use water from natural sources, from drilling performed in accordance with the law of mining, with the approval of the Ministry of Environment and under the supervision of the Ministry of Health. Medicinal water has to meet certain criteria of bacteriological and balneochemical tests in order to be considered curative.

Radon in large doses has a negative impact on health, and its adverse effects are connected with damaging enzymes and nucleic acids, which leads to the formation of neoplastic cells. Therefore, while using radon as a medicinal material one should have these effects in mind. The basis for a rational therapeutic effect of radon water is the so-called hypothesis of radiation hormesis. According to the theory, small doses of ionizing radiation activate life processes. Currently, there are no scientific reports explaining the mechanisms of radiation hormesis. Among the mechanisms at the level of the cell control system there are: stimulation of DNA repair processes, protein synthesis, activation of genes, production of stress proteins, detoxification of radicals, activation of membrane receptors, proliferation of splenocytes, and stimulation of the immune system, resulting in a lower risk of mutations or tumorigenesis. ${ }^{13}$

Radon water is specific water containing small quantities of unstable radioactive elements - radon and its decay products. The water is used in medicine when the content of radon exceeds $74 \mathrm{~Bq} / \mathrm{L}(2 \mathrm{nCi} / \mathrm{L})$, and when it meets operational and hygienic requirements. Radon is a chemical element formed in the process of disintegration of radioactive uranium and thorium. It is a noble, colorless and odorless gas, soluble in water, especially in weakly mineralized or acidified water. There are many radon isotopes, among which there is a precursor - radon-222 derived directly from radium-226 through alpha decay. The emitted alpha particles have low penetrating power, but high ionizing ability. The half-life is 3.8 days. The concentration of radon in natural conditions changes constantly throughout the day, as well as seasonally, in connection with precipitation. In the course of therapy, radon loss is also observed for technical reasons: while collecting water in reservoirs, transmission pipes, heating, chilling, and during intense exploitation. The decrease of radon content varies from $40 \%$ to $80 \%$. This large variation in the concentration of radon at a collecting place makes calculating the dose impossible; thus, it is not practiced. ${ }^{14}$ The absorption of radon in 95\% occurs in the lungs, whereas the excretion from the body occurs in $90 \%$ through the lungs and the remaining part - through the kidneys and skin. During radon baths, the absorption occurs mainly through inhalation, because radon and its derivatives accumulate in large quantities above the water. The lungs are exposed to radon to a significant extent due to the deposition of the degradation products in the alveoli. The radioactive residue is deposited on the skin surface and kept there for several hours. Radioactive decay in the body is very diverse and largely depends on the amount of body fat. 
In this process, the adrenal cortex, liver and muscles also play an important role. The anti-inflammatory, desensitizing and analgesic action of radon can be explained by the stimulation of the adrenal cortex and an increased production of steroid hormones. In the study, there was observed an increase in the concentrations of luteinizing hormone and growth hormone in serum, accompanied with an increase in cortisol, testosterone, estradiol, and estriol. Radon treatments improve peripheral circulation, reduce swelling, arthritis, tendon and muscle pain, and improve performance mobility. Lowered blood pressure, cholesterol and triglycerides, decreased red blood cell sedimentation reaction, increased hemoglobin levels and red blood cells, increased levels of ionized calcium, parathyroid hormone and calcitonin, accelerated removal of harmful products of metabolism were also observed. ${ }^{15-18}$

Spa (balneological) reaction is the body's adaptive response, created as a result of external factors (stimuli). It is a positive element of spa therapy, leading to metabolic changes, which are important for the body. Spa reaction is often the result of the use of a medicinal stimulus exceeding the body's compensation capacity. It is followed by excessive secretion of histamine, stimulation of the autonomic nervous system, burst of adrenal hormones by endocrine glands. The effect of these changes is an increase in the immune system and regeneration reactions. The mechanism of spa reaction is not yet fully examined. It is assumed, however, that in the abovementioned process it is mainly the autonomic nervous system that reacts to the applied stimulus.

The intensity of spa reaction depends on the type and quantity of natural medicinal materials used in the conducted study of radon water. The factors that determine the intensity of spa reaction are also the patients' age and state of health. In the image of spa reaction there may appear specific variable symptoms depending on the type of disease and non-specific ones, related to the reactivity of the organism treated with medical stimuli. These are mostly common ailments not previously observed in the patient. The symptoms can also be generalized in nature, have a local character and different levels of intensity. General symptoms include most commonly: increased erythrocyte sedimentation rate (ESR) and leukocyte number, an increase in body temperature, blood pressure, muscle pain, a decrease in overall physical performance. Local symptoms concern most commonly skin symptoms in the form of erythema, pruritus or urticaria. The reaction can affect even $70-80 \%$ of patients. The peak of spa reaction falls on the $2^{\text {nd }}-4^{\text {th }}$ day of the stay in a spa, increasing after about 7 days. On about the $14^{\text {th }}$ day of the stay at a sanatorium, the symptoms of spa reaction start to decrease. The exacerbation of the underlying disease and the activation of inflammatory reactions often appear. The clinical symptoms of the reaction are characterized with the intensity of muscle and joint pains, headaches, coronary pain, swelling of joints, loss of appetite, fatigue, irritability, depression, and sleep disorders. A characteristic feature of spa reaction is its division into phases: the initial phase I (lasts from 2 to 7 days) - labile reactions; the ergotrop phase II (lasts for about 1 week) - a period of an increased response; the trophop phase III (lasts until the end of treatment) - a relief reaction, a period of recovery and adaptation.

In phase III, the clinical improvement is achieved, often noticeable especially after returning home for a period of 8-10 months. Spa reaction usually occurs in patients who have used sulfur or radon baths and holistic mud peloid treatments. During the severe phase of spa reaction, it is advisable to reduce the intensity of the medicine stimulus, and sometimes it is recommended to temporarily discontinue the treatment. ${ }^{19,20}$

The aim of the study was to evaluate the changes in the scope of the integrated antioxidant system of the body in the course of radon therapy in terms of spa reaction.

\section{Material and methods}

The study was conducted in the health resort ŚwieradówZdrój. The observation included patients undergoing treatment in the spa within 21-day sanatorium stays. Venous blood was collected from patients before the treatment and after 18 days of treatment in the spa. Heparin plasma was used. To evaluate the TAS, a standard colorimetric assay by Randox Laboratories Ltd. (Warszawa, Polska) was applied. The obtained compound was characterized by a relatively stable blue-green color denoted with the use of light having a wavelength of $600 \mathrm{~nm}$. The presence of antioxidants in the sample weakens in the emergence of color in proportion to their concentration. The reference range of plasma in the sample was $1.30-1.77 \mathrm{mmol} / \mathrm{L}$ and it was determined for the European working population. The reference range is variable depending on genetic factors, and it is also characterized by local variability. The studies were non-randomized. The study group consisted of 35 patients with joints and spine pains caused by osteoarthritis or a discectomy. The age of patients ranged from 47 to 63 years. The mean age of patients was 56.5 years. Among the respondents there were 23 women and 11 men. The essential criteria for the selection of patients were the presence of degenerative joints and/or disc disease, the age range of $45-65$ years, the consent to participate in the study, and no contraindications to comprehensive treatment in the spa. The exclusion criteria were the lack of consent to participate in the research, age below 45 years and over 65 years, the presence of diseases constituting a contraindication to treatment (compatible with a standard list of indications and contraindications to spa therapy), and the presence of metabolic diseases. The patients were on a normal or light diet dominated by dishes 
prepared with low fat content. Both diets were standard calorie diets and there were no vitamin supplements used. Medicinal radon water was used in the therapy. Among the types of treatments applied there were: comprehensive radon bath - at a temperature of $37^{\circ} \mathrm{C}$, with a duration of $15 \mathrm{~min}$, the treatments performed every other day; radon inhalations - at a temperature of $37^{\circ} \mathrm{C}$, with a duration of $15 \mathrm{~min}$, the treatments performed every other day. Baths and inhalations were performed interchangeably and the total number of radon treatments during 1 stay was 15 . In addition, the following forms of therapy were used: kinesitherapy - with a duration of 30-45 min; physiotherapy - in limited extent due to the possibility of activating oxidation processes. The conducted study involved curative radon water from ŚwieradówZdrój that has been used in treatments for more than 100 years. The natural water with low mineralized content plays a major therapeutic role with the parameters of $\mathrm{Rn} 303.1-441.5 \mathrm{~Bq} / \mathrm{L}$. In the treatment rooms (an inhalation room, cabins with baths and a swimming pool), the alpha radiation was $184.4-450.0 \mathrm{MeV}$. This evaluation allows one to determine the exposure of the patient. In contrast, the calculation of the absorbed dose of radiation is not carried out, because it is a variable value. It depends both on the body anatomy, especially the fat content and the surface of absorption, and the presence of some diseases, as well as on radon loss dependent on its exploitation, as mentioned in the Introduction. The measurements were performed in a room on a daily basis with the use of certified detectors. The measurements were analyzed every 3 months at the Institute of Occupational Medicine in the Department of Radiation Protection in Łódź.

The study was designed with the participation of the control group. The control group consisted of 14 people selected from the employees of the spa, including 9 women and 5 men, aged from 50 to 62 years, with the average age of 54.2 years. The control group included persons suffering from osteoarthritis of the musculoskeletal system who were not given treatments with radon. The essential criteria in the selection were the presence of degenerative joints and disc disease, the age range of 45-65 years, the consent to participate in the research, and lack of diseases which are a contraindication to treatment. The exclusion criteria were the lack of consent to participate in research, age below 45 years and over 65 years and the presence of diseases which constitute a contraindication to treatment. The selection and exclusion criteria in the control group were the same as in the study group.

The study was conducted within the framework of the Polish Radon Cluster. The initiative Polish Radon Cluster was incorporated on 12 November, 2014. It is an organization which unites Polish radon spas that offer radon treatments on a large scale. Among the partners of the Radon Polish Cluster there are: Wroclaw Medical University, the Association Forum of Local
Activity and Sudecki Business Incubator, ŚwieradówCzerniawa Spa Ltd. - PGU Group, Lądek-Długopole SA Spa. The mission of the Polish Radon Cluster is to promote the use of the unique therapeutic and rehabilitation potential, which is at the disposal of spa management in mining areas, with the deposits of radon water, in the form of a voluntary agreement of entrepreneurs, individuals, legal entities, and research groups. The aim and the initiative of the Cluster relies in creating in Poland, particularly in the region of Lower Silesia, a modern national center for radon treatment based on the results of research and know-how of the members of the Polish Radon Cluster.

The study was approved by the Bioethics Committee of Wroclaw Medical University, Poland (No. 135/2015). A written consent was obtained from the President of the Swieradów-Czerniawa Spa and individual written consents were also obtained from the patients; they were prepared in accordance with the model recommended by the Bioethical Committee of Wroclaw Medical University. The documentation is held by the authors of the work.

For statistical analysis, STATISTICA v. 12 program (Randox Laboratories Polska, sp. z o.o. Warszawa, Poland) was used. Arithmetic means (a measure of the position), standard deviations (a measure of variability) and a range of variability (extreme values) were calculated for measurable variables. All the tested quantitative variables were checked with the Shapiro-Wilk test to determine the type of distribution. The comparison between the results of measurements in each group was performed using the Friedman ANOVA and post-hoc test. For all the comparisons, the level of $\alpha=0.05$ was assumed and the obtained p-values were rounded to 4 decimal places.

\section{Results}

In the study group, an increase in the level of TAS in trials II and III was noted. In the control group, in test II, the level of TAS increased, whereas in trail III, a decrease of TAS below the output value was noted. The Friedman ANOVA analysis of variance showed statistically significant changes in the test group and a slightly lower result in the control group. A multiple comparison with the use of the Friedman post-hoc test ANOVA of measurements I and II, II and III showed a statistically significant change in relation to the study group (an increase in the TAS concentration). In the case of the control group, an increase in the concentration of TAS in test II was statistically significant. In contrast, the comparison of measurements II and III showed a decrease in the TAS concentration and exhibited the characteristics of significance. The p-value in the comparison of measurement I and III for the 2 groups did not meet the target level of significance (Table 1, Fig. 1). 
Table 1. Comparison of results in both groups

\begin{tabular}{|c|c|c|c|c|c|c|c|c|}
\hline Parameters & TAS [mmol/L] & $\mathrm{N}$ & $\bar{x}$ & Min & $\operatorname{Max}$ & $\mathrm{SD}$ & $p$-value* & $p$-value** \\
\hline \multirow{3}{*}{ Study group } & measurement I & 35 & 1.82 & 1.55 & 2.06 & 0.14 & \multirow{3}{*}{0.0000} & \multirow{3}{*}{$\begin{array}{l}\text { I vs } \|-p=0.0001 \\
\text { I vs } \| \mid-p=0.3788 \\
\| \text { vs }||-p=0.0001\end{array}$} \\
\hline & measurement II & 35 & 2.07 & 1.80 & 2.53 & 0.20 & & \\
\hline & measurement III & 35 & 1.87 & 1.71 & 2.13 & 0.12 & & \\
\hline \multirow{3}{*}{ Control group } & measurement I & 15 & 1.83 & 1.65 & 2.09 & 0.11 & \multirow{3}{*}{0.0013} & \multirow{3}{*}{$\begin{array}{l}\text { I vs }||-p=0.0071 \\
\text { I vs III - } p=0.9444 \\
\text { II vs III-p }=0.0030\end{array}$} \\
\hline & measurement II & 15 & 1.97 & 1.81 & 2.21 & 0.12 & & \\
\hline & measurement III & 15 & 1.81 & 1.62 & 2.10 & 0.14 & & \\
\hline
\end{tabular}

$\mathrm{N}$ - number of trials; $\bar{x}$ - arithmetic mean (a measure of position); Min - minimum; Max - maximum; SD - standard deviation (a measure of variability); * the Friedman analysis of variance (ANOVA); ${ }^{* *}$ multiple comparisons in the Friedman post-hoc ANOVA.

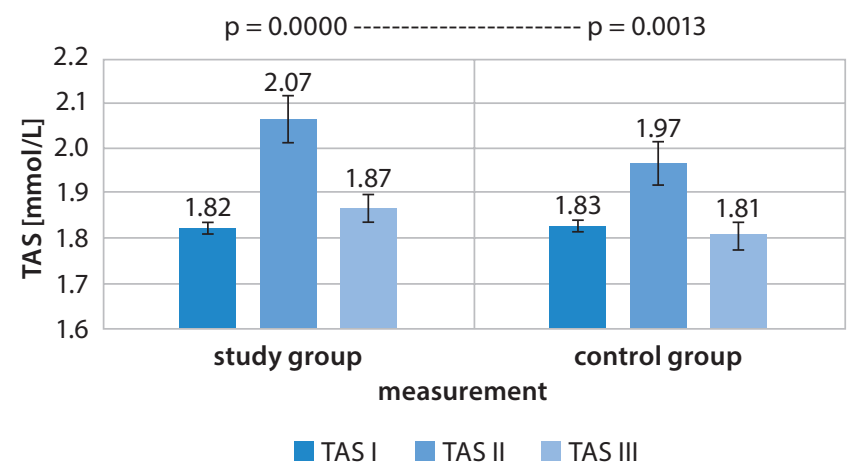

Fig. 1. Comparison of the means in both groups for 3 measurements variance analysis (Friedman ANOVA)

\section{Discussion}

In the study group, an increase in the TAS concentration after the treatment was noted, whereas in the control group, a decrease in TAS of the system was noted, which can indicate and determine the effectiveness of the spa therapy. In the conducted observation, an important issue was the assessment of the level of TAS on the $5^{\text {th }}$ day of the treatment (measurement II). In both groups, an increase in TAS of the system was found. In the study group, there was a statistically significant change in the concentration of TAS ( $\mathrm{p}=0.0001)$, whereas in the control group it was $\mathrm{p}=0.007$ (Table 1 ). The obtained TAS results may indicate the sensitivity of the antioxidant system to radon therapy. The increase in the TAS concentration in the study group was due to the activation of the antioxidant system of the body as a result of the conducted therapy. However, in the control group, the increase in TAS on the $5^{\text {th }}$ day is unclear, and the change in value is less significant. In addition, the end result indicates a decrease in the concentration of TAS in the control group, which could ultimately indicate the lack of a factor stimulating the antioxidant system. The activation of the antioxidant system may be affected by a number of metabolic changes, which were discussed in the Introduction. Currently, it is difficult to assess the extent to which ionizing radiation stimulates the antioxidant system. There are not many publications that would match the results of the conducted observation. Most articles published in the $60 \mathrm{~s}, 70 \mathrm{~s}$ and $80 \mathrm{~s}$ of the $20^{\text {th }}$ century, as well as studies on animals conducted in Japan, discussed the effects of radon therapy in very general terms. The studies demonstrated mainly the analgesic effects, decreased swelling, improvement in physical fitness. Years later, the studies on the subject matter showed that radon decreased the release of ROS in patients with Bechterew's disease and acted as an anti-proliferative agent to tumor cells, stimulating, i.a., the activity of superoxide dismutase. ${ }^{21-25}$ Most of the publications discuss the health effects of ionizing radiation exposure, but only in reference to a specific case. Some of the reports refer to the studies of the populations living in areas with naturally high radiation or populations inhabiting the areas after a nuclear explosion, and those after occupational exposures. These studies showed that low doses of radiation had not only a neutral but also a positive impact on health. ${ }^{26}$ Spa reaction is an important criterion of the body's reactivity to medicinal stimuli in the course of therapeutic spa treatment. When evaluating the results, it can be assumed that the human body receives a balneological stimulus as a factor causing free radical reactions. The process reveals a variety of symptoms and conditions. In a further step, by the accumulation of stimuli one can observe a change in the metabolism and an activation of immune reactions. It is followed by an increase in the production of antioxidants. The end result is a higher level of TAS of the body, the start of the adaptation and regeneration processes, and the clinical improvement after the treatment. The use of balneological stimuli should be individualized and adjusted to the adaptive capacity of the patient. The use of inappropriately strong stimuli may cause adverse effects in the form of more or less serious disequilibrium in homeostasis. On the other hand, the use of a medicinal stimulus with inadequately low intensity will not cause the expected results in terms of improving the health status of the patient. Spa reaction as a specific body response finally resulting in the improvement of the patient status (which occurs in the last phase of spa treatment) may express a controlled disturbance of homeostasis through a complex, lasting several days, activity with an exposure to the stimulus. The stimuli used in a spa express the same 
character as adaptive stress. In the case of radon water used in the study, as a result of ionizing radiation (stimulus), used with specified time intervals, there was observed an increase in the resistance to a stress-applied stimulus. The result of these reactions will be adaptation, body hardening and habituation. A possible positive effect is an increase of the body's immune reactions due to little known mechanisms of temporary, functional or morphological changes dependent also on the stimulation of the autonomic nervous system. These mechanisms may give rise to positive changes determining the systemic improvement after the spa treatment. ${ }^{27,28}$ In the case of radon water with low doses of radiation, it is relatively difficult to find statistically reliable material. Much of the work in which the conclusions are drawn is not based on the observations of the effects of radiation, but on their absence. A big problem is the absorbed dose of radiation, the scope of systemic changes and the loss of radiation during radon water distribution. The main element believed to activate the transformation are low doses of absorbed ionizing radiation used in accordance with the theory of radiation hormesis. Irradiation of a large mass of tissue brings more pronounced systemic effects than focusing all radiation energy on an isolated part of the body. A possible positive effect is habituation. ${ }^{29,30}$ It can be assumed that an increase in immunity, physical fitness, pain relief, the normalization of blood pressure, a decreased heart rate are the effects of small doses of ionizing radiation, resulting from the stimulation of the antioxidant system. A clear indicator of such changes is the increase in TAS of the body in the study group after the treatment.

The assessment of the positive impact of radon therapy and other forms of spa therapy treatment in reference to the phenomenon of spa reaction requires conducting multidisciplinary research with a specific explanation of the significance of the phenomenon of radiation hormesis. It is desirable to design randomized studies conducted in large groups of patients.

\section{Conclusions}

A beneficial effect of radon treatments on the growth of TAS in the body of the patients treated in the spa was demonstrated.

The increase in the TAS concentration on the $5^{\text {th }}$ day of treatment may indicate the relationship between these changes and the phenomenon of spa response.

The changes are a result of low doses of ionizing radiation originating from radon dissolved in medicinal water used in the course of the therapy.

\section{References}

1. Gorzenkowicz J, Wojtkowiak D, Podhajska AJ. Reactive oxygen species as signal molecules. Pol J Cosmetol. 2002;5(2):90-106.

2. Wiktorowska-Owczarek A, Nowak J. Pathogenesis and prevention of AMD: The role of oxidative stress and antioxidants. Adv Hig Exp Med (online). 2010;64:333-343.
3. Pedzik A, Paradowski M, Rysz J. Oxidative stress and pathological phenomena in the regime. Diagn Lab. 2008;44:1-7.

4. Opara E C. Oxidative stress. Dis Mon. 2006;52(2):183-198.

5. Roessner A, Kuester D, Malfertheiner P, Schneider-Sock R. Oxidative stress in ulcerative colitis-associated carcinogenesis. Pathol Res Pract. 2008;204:511-524.

6. Puzanowska-Tarasierwicz H, Kuźmicka L, Tarasiewicz L. Influence of reactive nitrogen and oxygen species on human organism. Pol Med J. 2009;27:162:496-498.

7. Makowski M, Grzela T, Niderla J, et al. Inhibition in cyclooxygenase-2 indirectly potentiates antitumor effects of photodynamic therapy in mice. Clin Cancer Res. 2003;9:5417-5422.

8. Ryszawa N, Kawczyńska-Drożdż A, Pryjma J, et al. Effects of novel plant antioxidants on platelet superoxide production and aggregation in arteriosclerosis. J Physiol Pharmacol. 2006;57:611-626.

9. Czajka A. Reactive oxygen species and mechanisms of body protection. JMS. 2006;75(6):582-586.

10. Duarte TL, Lunec J. When is an antioxidant not an antioxidant? A review of novel actions and reactions of vitamin C. Free Radic Res. 2005;39:671-686.

11. Rahman K. Studies on free radicals, antioxidants, and co-factors. Clin Interv Aging. 2007;2:563-567.

12. Zabłocka A, Janusz M. Two faces of free radicals. Adv Hig Exp Med. 2008;62:118-124.

13. Kraska A, Bilski B. Exposure of healthcare personnel to ionizing radiation in the light radiation hormesis hypothesis. Medycyna Pracy. 2012;63(3):371-376.

14. Kochański W, Minta P, Kochański M, Trzewikowska I. Problems dosimetry radon therapy. Pol Baln. 2004;46(1/2):49-57.

15. Demczyszak I, Perkowska K, Wrzosek Z, Sutkowska E. Alpha therapy application in the health resort environment. Pol Baln. 2009;51(1):57-58.

16. Zdrojewicz Z, Belowska-Bień K. Estimating testosterone concentrations in male patients exposed to radon media. Adv Hig Exp Med. 2004;513(2):267-272.

17. Zdrojewicz Z, Belowska-Bień K. Radon and ionizing radiation in the human body. Adv Hig Exp Med. 2004;58,150-157.

18. Belowska-Bień K, Zdrojewicz Z. Influence of radon media treatment on secretion of prolactin. Adv Hig Exp Med. 2005;14(5):132-138.

19. Drobnik J, Malcewicz M, Józefowski P, Kurpas D, Steciwko A. Health resort medicine: Contemporary resort health care in Poland. Familiy Medicine \& Primary Care Reviev. 2011;13(1):103-108.

20. Samborski W, Ponikowska I. Rheumatic diseases: Medical standards in health resort therapy. Balneol Pol. 2005;47(1-2):14-23.

21. Ishimori Y, Mitsunobu F, Yamaoka K, Tanooka H, Kataoka T, Sakoda A. Performance of the first Japanese large-scale facility for radon inhalation experiments with small animals. Radiat Prot Dosimetry. 2011;7:146(1-3):31-33.

22. Sakoda A, Ishimori Y, Yamaoka K, Kataoka T, Mitsunobu F. Absorbed doses of lungs from radon retained in airway lumens of mice and rats. Radiat Environ Biophys. 2013;52:389-395.

23. Reinisch N. Reduction der Sauerstoffradikalfreisetzung aus Neutophilen. In: Deetjen P, Falkenbach A, eds. Radon und Gesundheit. Frankfurt am Main: Pert Lang, Europäischer Verlag der Wissenschaften; 1999:75-82.

24. Soto J. Radon effects at cellular and mollecular levels. In: Deetjen $P$, Falkenbach A, eds. Radon und Gesundheit. Frankfurt am Main: Pert Lang, Europäischer Verlag der Wissenschaften; 1999:63-66.

25. Yamaoka K, Edamatsu R, Mori A. Increased SOD activities and decreased lipid peroxide levels induced by low dose $\mathrm{X}$ irradiation in rat organs. Free Radic Biol Med. 1991;11:299-306.

26. Vaiserman A. Radiation hormesis: Historical perspective and implications for low-dose cancer risk assessment. Dose Response. 2010;8:172-191.

27. Kalmus P, Szynkowska L. Obserwations of the adaptive reactions in patients with inflammatory rheumatic diseases during health resort therapy. Acta Baln. 2015;57:97-105.

28. Mothersill C, Seymour C. Radiation-induced bystander effects: Evidence for adaptive response to low dose exposure? Dose Response. 2006;4(4):283-290.

29. Cuttler J, Pollycove M. Nuclear energy and health and the benefits of low-doses radiation hormesis. Dose Response. 2009;7(1):52-89.

30. Strzelczyk J, Potter W, Zdrojewicz Z. Rad-by-Rad (Bit-by-Bit):Triumph of evidence over activities fostering fear of radiogenic cancers at low doses. Dose Response. 2007;5(4):275-283. 